\title{
Identification of novel molecular markers for detection of gastric cancer cells in the peripheral blood circulation using genome-wide microarray analysis
}

\author{
NOBUYUKI MATSUMURA, HITOSHI ZEMBUTSU, KOJI YAMAGUCHI, KAZUAKI SASAKI, \\ TETSUHIRO TSURUMA, TOSHIHIKO NISHIDATE, RYUICHI DENNO and KOICHI HIRATA
}

First Department of Surgery, Sapporo Medical University, School of Medicine, Hokkaido 060-0061, Japan

Received March 8, 2011; Accepted April 5, 2011

DOI: $10.3892 / \mathrm{etm} .2011 .252$

\begin{abstract}
Although metastasis or relapse is a leading cause of death for patients with gastric cancer, the hematogenous spread of cancer cells remains undetected at the time of initial therapy. The development of novel diagnostic molecular marker(s) to detect circulating gastric cancer cells is an issue of great clinical importance. We obtained peripheral blood samples from 10 patients with gastric cancer who underwent laparotomy and 4 healthy volunteers. Microarray analysis consisting of 30,000 genes or ESTs was carried out using eight gastric cancer tissues and normal gastric mucosae. We selected 53 genes up-regulated in gastric cancer compared to normal gastric mucosae from our microarray data set, and, among these, identified five candidate marker genes (TSPAN8, EPCAM, MMP12, MMP7 and REG3A) which were not expressed in peripheral blood mononuclear cells (PBMCs) from 4 healthy volunteers. We further carried out semi-quantitative nested reverse transcription-polymerase chain reaction (RT-PCR) for HRHI, EGFR, CK2O and CEA in addition to the five newly identified genes using PBMCs of patients with gastric cancer, and found that expression of one or more genes out of the nine was detected in $80 \%$ of the patients with gastric cancer. Moreover, the numbers of genes expressed in PBMCs were $\leq 2$ and $\geq 2$ in all vascular invasion-negative cases and in 5 of 6 positive cases, respectively, showing significant differences between the two groups $(\mathrm{P}=0.041)$. Nested RT-PCR analysis for the set of nine marker genes using PBMCs may provide the potential for detection of circulating gastric cancer cells prior to metastasis formation in other organs.
\end{abstract}

Correspondence to: Professor Koichi Hirata, First Department of Surgery, Sapporo Medical University, School of Medicine, Hokkaido 060-0061, Japan

E-mail: hitoshi@rc4.so-net.ne.jp

Key words: microarray, gastric cancer, molecular marker, nested RT-PCR, peripheral blood

\section{Introduction}

Gastric cancer causes approximately 800,000 deaths worldwide per year and is still one of the leading causes of cancer-related death in the world (1). Most gastric cancers at an early stage can be cured by surgical resection; however, patients with advanced gastric cancers have worse prognosis than those with early stage disease (2). Although metastasis or relapse is the main cause of death for patients with gastric cancer (3), the hematogenous spread of malignant cells remains undetected at the time of initial therapy. During the development of cancer, tumor cells may detach from the primary tumor and disseminate into the lymph system and/ or blood circulation, and grow in the bone marrow, liver, kidney and other organs, which is called micrometastasis (4). Micrometastasis is barely detected by routine biochemical and histopathological assays or graphical methods, such as X-ray, CT and MRI (3). Detection of circulating tumor cells at the mRNA level [reverse transcription-polymerase chain reaction (RT-PCR)] in blood samples of patients with cancer could serve as a unique and easy diagnostic tool to predict cancer recurrence and to monitor treatment effectiveness (5-7). However, molecular marker(s) that detect circulating gastric cancer cells for routine clinical use have not yet been identified. Hence, the development of novel diagnostic molecular marker(s) to detect circulating gastric cancer cells is an issue of great clinical importance.

Carcinoembryonic antigen (CEA) is a well-known tumor marker and has been used to detect small amounts of adenocarcinoma cells in the blood, peritoneal wash or other body fluids (8-12). However, the expression of CEA mRNA is not specific to cancer cells and often produces false-positive results (13). Profiling of gene expression patterns on genome-wide microarrays enables investigators to perform comprehensive characterization of molecular activities in cancer cells (14-17). Systematic analysis of expression levels for thousands of genes is also a useful approach for identifying molecular markers to detect small amounts of circulating cancer cells (18). In this study, we identified genes whose expression had been altered during gastric carcinogenesis using genome-wide information obtained from 8 cases on a microarray consisting of 30,000 transcribed elements. Based on the results of the microarray 
Table I. Characteristics of patients included in the nested RT-PCR analysis of PBMCs.

\begin{tabular}{lc} 
Parameters & No. of patients \\
\hline Gender (male:female) & $5: 5$ \\
Age range (average), in years & $41-82(61.9)$ \\
Depth of tumor invasion (T1:T2:T3:T4) & $1: 6: 2: 1$ \\
Lymph node metastasis (N0:N1:N2:N3) & $4: 3: 2: 1$ \\
Distant metastasis (M0:M1) & $10: 0$ \\
Liver metastasis (H0:H1) & $10: 0$ \\
Peritoneal metastasis (P0:P1) & $9: 1$ \\
Peritoneal lavage cytology (CY0:CY1) & $9: 1$ \\
Stage (I:II:III:IV) & $4: 1: 2: 3$ \\
Lymphatic invasion (ly0:ly1-3) & $2: 8$ \\
Vessel invasion (v0:v1-3) & $4: 6$ \\
\hline
\end{tabular}

assay, we identified five candidate genes for the specific detection of circulating gastric cancer cells at the mRNA level. We suggest that such information may lead ultimately to improve the prognosis of patients with gastric cancers.

\section{Materials and methods}

Blood and tissue samples. Blood samples were obtained from 10 patients with gastric cancer who underwent laparotomy and 4 healthy volunteers after obtaining informed consent. Heparinized blood samples $(5 \mathrm{ml})$ from the 10 patients with gastric cancer were obtained from a peripheral artery through a catheter used for monitoring arterial blood pressure during surgical operation. Peripheral venous blood was obtained from 4 healthy volunteers for control after discarding the initial $10 \mathrm{ml}$ of blood to protect the mixture from epithelial cells. Clinicopathological characteristics of the 10 patients are shown in Table I. Clinical stage of each patient was judged according to the Union for International Cancer Control (UICC) TNM classification. Among the 10 patients with gastric cancer, 8 primary gastric cancer tissues and corresponding non-cancerous gastric mucosae from surgically resected tissues were obtained at Sapporo Medical University and Douto Hospital after each patient had provided informed consent. The samples that had been confirmed histologically as gastric adenocarcinoma were used for microarray study. These samples were immediately frozen and stored at $-80^{\circ} \mathrm{C}$. All cancer tissues were obtained from the margin of the tumor mass, while non-cancerous tissues were obtained from corresponding normal mucosae of the same stomach. This study was approved by the Ethics Committee of Sapporo Medical University, School of Medicine, Hokkaido, Japan.

RNA extraction of blood samples. We prepared peripheral blood mononuclear cells (PBMCs) using Ficoll (Amersham Biosciences, Buckinghamshire, UK) and extracted total RNA using TRIzol (Invitrogen, Inc., Carlsbad, CA, USA) according to the manufacturer's instructions. Before the synthesis of cDNA, deoxyribonuclease I (DNase I) (Nippon Gene, Japan) was added to each sample of total RNA according to the manufacturer's instructions.

Analysis of microarray. Total RNA was extracted from each gastric tissue using TRIzol according to the manufacturer's instructions. To guarantee the quality of RNAs, total RNA extracted from the residual tissue of each case was electrophoresed on a denaturing agarose gel, and the quality was confirmed by the presence of rRNA bands. After treatment with DNase I, T7-based RNA amplification was carried out as described previously with a few modifications (19). Using $5 \mu \mathrm{g}$ of total RNA from each tissue sample as starting material, one round of amplification was performed; the amount of each amplified RNA (aRNA) was measured by a spectrophotometer. A mixture of normal gastric mucosae from 8 patients was prepared as a universal control and was amplified in the same manner; $2.5 \mu \mathrm{g}$ of aRNAs from each cancerous tissue and from the control was reversely transcribed in the presence of Cy5-dCTP and Cy3-dCTP, respectively (15). AceGene 30K-1 Chip Version (Hitachi Software Engineering Co., Japan) was used for microarray analysis. The procedures for hybridization, washing, photometric quantification of signal intensities of each spot and normalization of data were according to the manufacturer's instructions. To normalize the amount of mRNA between tumors and controls, the fluorescence intensities of Cy5 (gastric cancer) and Cy3 (control) for each target spot were adjusted so that the mean $\mathrm{Cy} 5 / \mathrm{Cy} 3$ ratio of 30,000 genes equaled 1 . Genes were categorized into three groups according to the cancer/normal ratio of their mean signal intensity: up-regulated (expression ratio $>5.0$ ), downregulated (expression ratio <0.2) and unchanged expression (expression ratio between 0.2 and 5.0).

Semi-quantitative $R T-P C R$. To validate the result of the microarray analysis, we examined the expression levels of the genes up-regulated in gastric cancer by means of semiquantitative RT-PCR analysis. Total RNAs ( $3 \mu \mathrm{g})$ extracted from each cancerous tissue and normal gastric mucosa were reversely transcribed for single-stranded cDNAs using oligo(dT)12-18 primer with Superscript II reverse transcriptase (Life Technologies, Inc.). Each single-stranded cDNA was diluted for subsequent PCR amplification. A housekeeping gene, GAPDH, served as the internal control. The PCR reaction was conducted at $95^{\circ} \mathrm{C}$ for $5 \mathrm{~min}$, and then for 30 cycles at $95^{\circ} \mathrm{C}$ for $30 \mathrm{sec}, 60^{\circ} \mathrm{C}$ for $30 \mathrm{sec}$ and $72^{\circ} \mathrm{C}$ for $1 \mathrm{~min}$ followed by $72^{\circ} \mathrm{C}$ for $10 \mathrm{~min}$, in the Gene Amp PCR System 9700 (Perkin-Elmer Applied Biosystems, Foster City, CA, USA).

Nested RT-PCR using blood samples. We performed nested RT-PCR using total RNAs extracted from PBMCs to accurately examine mRNA levels of the candidate marker genes. Initially, RT-PCR was carried out as described above. In nested RT-PCR, $1 \mathrm{ml}$ of the initial PCR product, $4 \mathrm{ml}$ of $10 \mathrm{X}$ PCR buffer, $200 \mathrm{mmol} / 1 \mathrm{dNTP}$ mixture, $0.2 \mathrm{mmol} / 1$ primers and 1 unit Taq DNA polymerase (Takara) were added to a 40-ml aliquot of the reaction mixture. The PCR reaction was conducted at $95^{\circ} \mathrm{C}$ for $5 \mathrm{~min}$, and then 30 cycles at $95^{\circ} \mathrm{C}$ for $30 \mathrm{sec}, 60^{\circ} \mathrm{C}$ for $30 \mathrm{sec}$ and $72^{\circ} \mathrm{C}$ for $1 \mathrm{~min}$ followed by $72^{\circ} \mathrm{C}$ for $10 \mathrm{~min}$, in the Gene Amp PCR System 9700 . The RT-PCR products were detected using $2 \%$ agarose gel 
Table II. Primer sequences for semi-quantitative nested RT-PCR.

\begin{tabular}{lll}
\hline Gene & \multicolumn{1}{c}{ Forward primer } & \multicolumn{1}{c}{ Reverse primer } \\
\hline TSPAN8 & 5'-TCAACTTCTTGTTCTGGCTATGT-3' & 5'-TATAGCTTTGGCATGGTCTCTGC-3' \\
EPCAM & 5'-TGATCCTGACTGCGATGAGAGC-3' & 5'-CAGCTTTCAATCACAAATCAGT-3' \\
$M M P 12$ & 5'-AACCAGCTCTCTGTGACCCCA-3' & 5'-TCCAAGGATGTTAGGAAGCAAC-3' \\
$R E G 3 A$ & 5'-TCTCTGGACGGCAGCTATGCG-3' & 5'-AATAAGACACAGTCACACCATAA-3' \\
HRH1 & 5'-GTATCTTGGATGCTGCTTTCCTG-3' & 5'-GTATGACAAAATGAAGAGACTGA-3' \\
$E G F R$ & 5'-TACAAGGCCGTACGACAACACT-3' & 5'-TCTGCTGTTCTTCTATGGTGCCT-3' \\
$C K 20$ & 5'-ATGTCCCCACGGTACTTACTCCC-3' & 5'-TCTTAACAATGCTGTAGGGGCTC-3' \\
$C E A$ & 5'-TGGATTTCAGTCGCAGA-3' & 5'-ATGTAGGGTTAGGTCATCAAAG-3' \\
& 5'-TTCTCCTGGTCTCTCAGCTGGG-3' & 5'-AATGCTTTAAGGAAGAAGCAA-3'
\end{tabular}

Table III. Genes up-regulated in advanced gastric cancer.

\begin{tabular}{|c|c|c|c|}
\hline No. & Accession no. & Gene symbol & Description \\
\hline 1 & NM_006507 & $R E G 1 B$ & Regenerating islet-derived $1 \beta$ (pancreatic stone protein, pancreatic thread protein) \\
\hline 2 & NM_004577 & PSPH & Phosphoserine phosphatase \\
\hline 3 & NM_138938 & REG3A & Regenerating islet-derived $3 \alpha$ \\
\hline 4 & NM_014471 & SPINK4 & Serine peptidase inhibitor, Kazal type 4 \\
\hline 5 & NM_001105249 & TMC5 & Transmembrane channel-like 5 \\
\hline 6 & NM_002426 & MMP12 & Matrix metallopeptidase 12 (macrophage elastase) \\
\hline 7 & NM_002423 & $M M P 7$ & Matrix metallopeptidase 7 (matrilysin, uterine) \\
\hline 8 & NM_002483 & CEACAM6 & $\begin{array}{l}\text { Carcinoembryonic antigen-related cell adhesion } \\
\text { molecule } 6 \text { (non-specific cross reacting antigen) }\end{array}$ \\
\hline 9 & NM_001786 & $C D C 2$ & Cell division cycle $2, \mathrm{G} 1$ to $\mathrm{S}$ and $\mathrm{G} 2$ to $\mathrm{M}$ \\
\hline 10 & NM_000582 & $S P P 1$ & $\begin{array}{l}\text { Secreted phosphoprotein } 1 \text { (osteopontin, bone sialoprotein I, } \\
\text { early T-lymphocyte activation } 1 \text { ) }\end{array}$ \\
\hline 11 & NM_004751 & GCNT3 & Glucosaminyl (N-acetyl) transferase 3 , mucin type \\
\hline 12 & NM_000574 & CD55 & CD55 molecule, decay accelerating factor for complement (Cromer blood group) \\
\hline 13 & NM_002443 & $M S M B$ & Microseminoprotein, $\beta$ \\
\hline 14 & NM_004336 & $B U B 1$ & UB1 budding uninhibited by benzimidazoles 1 homolog (yeast) \\
\hline 15 & NM_015017 & USP33 & Ubiquitin-specific peptidase 33 \\
\hline 16 & NM_004591 & CCL2O & Chemokine (C-C motif) ligand 20 \\
\hline 17 & NM_017633 & FAM46A & Family with sequence similarity 46 , member A \\
\hline 18 & NM_000088 & COLIA1 & Collagen, type I, $\alpha 1$ \\
\hline 19 & XR_017717 & ADAMTSL3 & ADAMTS-like 3 \\
\hline 20 & NM_138938 & $R E G 3 A$ & Regenerating islet-derived $3 \alpha$ \\
\hline 21 & NM_017934 & PHIP & Pleckstrin homology domain interacting protein \\
\hline 22 & XR_016124 & & Similar to p21-activated kinase 2 \\
\hline 23 & NM_006398 & $U B D$ & Ubiquitin D \\
\hline 24 & NM_002358 & $M A D 2 L 1$ & MAD2 mitotic arrest deficient-like 1 (yeast) \\
\hline 25 & NM_002483 & CEАCAM6 & $\begin{array}{l}\text { Carcinoembryonic antigen-related cell adhesion } \\
\text { molecule } 6 \text { (non-specific cross-reacting antigen) }\end{array}$ \\
\hline 26 & NM_173164 & IPO9 & Importin 9 \\
\hline 27 & NM_003014 & SFRP4 & Secreted frizzled-related protein 4 \\
\hline 28 & NM_004616 & TSPAN8 & Tetraspanin 8 \\
\hline 29 & NM_002354 & EPCAM & Epithelial cell adhesion molecule \\
\hline 30 & NM_006498 & $L G A L S 2$ & Lectin, galactoside-binding, soluble, 2 \\
\hline 31 & NM_002372 & $M A N 2 A 1$ & Mannosidase, $\alpha$, class $2 \mathrm{~A}$, member 1 \\
\hline 32 & NM_003937 & $K Y N U$ & Kynureninase (L-kynurenine hydrolase) \\
\hline 33 & NM_003821 & $R I P K 2$ & Receptor-interacting serine-threonine kinase 2 \\
\hline 34 & NM_00108039 & ITGA7 & Integrin, $\alpha 7$ \\
\hline 35 & NM_000670 & ADH4 & Alcohol dehydrogenase 4 (class II), $\pi$ polypeptide \\
\hline
\end{tabular}


Table III. Continued.

\begin{tabular}{|c|c|c|c|}
\hline No. & Accession no. & Gene symbol & Description \\
\hline 36 & NM_014314 & $D D X 58$ & DEAD (Asp-Glu-Ala-Asp) box polypeptide 58 \\
\hline 37 & NM_006418 & OLFM4 & Olfactomedin 4 \\
\hline 38 & NM_198187.3 & ASTN2 & Astrotactin 2 \\
\hline 39 & NM_015364 & LY96 & Lymphocyte antigen 96 \\
\hline 40 & NM_000574 & CD55 & CD55 molecule, decay accelerating factor for complement (Cromer blood group) \\
\hline 41 & NM_018964 & SLC37A1 & Solute carrier family 37 (glycerol-3-phosphate transporter), member 1 \\
\hline 42 & NM_018455 & CENPN & Centromere protein $\mathrm{N}$ \\
\hline 43 & NM_001710 & $C F B$ & Complement factor B \\
\hline 44 & NM_033020 & TRIM33 & Tripartite motif-containing 33 \\
\hline 45 & NM_003090 & SNRPAl & Small nuclear ribonucleoprotein polypeptide A\&apos; \\
\hline 46 & NM_000373 & $U M P S$ & $\begin{array}{l}\text { Uridine monophosphate synthetase (orotate phosphoribosyl transferase and } \\
\text { orotidine-5\&apos;-decarboxylase) }\end{array}$ \\
\hline 47 & NM_144584 & Clorf59 & Chromosome 1 open reading frame 59 \\
\hline 48 & NM_052916.2 & $R N F 157$ & Ring finger protein 157 \\
\hline 49 & NM_006332 & IFI30 & Interferon, $\gamma$-inducible protein 30 \\
\hline 50 & NM_002416 & CXCL9 & Chemokine (C-X-C motif) ligand 9 \\
\hline 51 & NM_001017402 & $L A M B 3$ & Laminin, $\beta 3$ \\
\hline 52 & NM_004701 & $C C N B 2$ & Cyclin B2 \\
\hline 53 & NM_194463 & $R N F 128$ & Ring finger protein 128 \\
\hline
\end{tabular}

electrophoresis. The primer sequences are summarized in Table II.

\section{Results}

Identification of up-or down-regulated genes in the gastric cancers. We extracted RNAs from eight primary gastric cancer tissues and corresponding normal gastric mucosae as control, and carried out gene expression analysis using a microarray consisting of 30,000 genes or ESTs. We then selected genes from our data set according to the criterion that the cancer/ normal ratio of the mean signal intensity of a given gene was $>5.0$ or $<0.2$, and 53 genes were identified as up-regulated and 123 genes as down-regulated in the gastric cancer tissues compared to the normal gastric mucosa (Tables III and IV). The up-regulated genes represented a variety of functions, including genes associated with signal-transduction pathways (SFRP4 and TSPAN8), genes encoding transcription factors (TRIM33), genes involved in various metabolic pathways (ADH4, USP33, RNF128, MAN2A1, UBD and GCNT3), apoptosis (SPP1 and RIPK2), chemokines (CCL20), DNA replication and recombination (SNRPAI), cell adhesion and cytoskeleton ( $L A M B 3, E P C A M, M M P 7$ and COL1A1), cellcell signaling (CEACAM6 and $C X C L 9)$, cell cycle $(C D C 2$, $B U B 1$ and $C C N B 2)$, cell proliferation (REGIB and REG3A), or other functions (SPINK4, TMC5, LGALS2, KYNU, DDX58, LY96, UMPS and RNF157).

On the other hand, the down-regulated genes included those associated with various metabolic pathways ( $C K M$, $A R S D$ and $B G N)$, small molecule transport (ACCN3, ATP6VOA1 and SFXN1), signal transduction (FLT4, NPBWR2, CSF3R and FZD8), cell cycle regulation (TBCID1, DLG5, GAS2L1, CDC34 and SH2B1), cell adhesion (RAPH1,
CLDN10, BCAN, CD164 and JUP), transcription factors (LDB1, CLOCK, GCM1, BRF1, TCF3, PML and AIRE), cellcell signaling (PGD, S100A9, CCL13 and RIMS1) or other functions.

Identification of candidate genes as molecular markers for the detection of circulating gastric cancer cells in human peripheral blood. Of the 53 genes that were up-regulated in the gastric cancer compared to the normal gastric tissues, we identified five candidate marker genes [tetraspanin 8 (TSPAN8), epithelial cell adhesion molecule (EPCAM), matrix metallopeptidase 12 (MMP12), matrix metallopeptidase 7 $(M M P 7)$ and regenerating islet-derived $3 \alpha(R E G 3 A)$ ] for the detection of circulating gastric cancer cells in peripheral blood in accordance with the following criteria: i) no or weak expression in human normal tissues in the published database (20), ii) no expression in PBMCs from 4 healthy volunteers by nested RT-PCR. In addition to the above five newly identified genes, we analyzed histamine receptor H1 (HRH1) since a previous study reported that this gene was overexpressed in gastric cancer cells, and the expression of this gene satisfied the above criteria (21). Moreover, three candidate marker genes [keratin $20(C K 20)$, epidermal growth factor receptor $(E G F R)$ and carcinoembryonic antigen $(C E A)]$, which have been reported to be promising markers for the detection of cancer cells, were further analyzed $(11,13,22,23)$.

Association of the expression of the nine marker genes for the detection of circulating gastric cancer cells with clinicopathological parameters by nested RT-PCR. We carried out semi-quantitative nested RT-PCR analysis of the nine candidate marker genes for the detection of circulating cancer cells using PBMCs of patients with gastric cancer. Of the nine 
Table IV. Genes down-regulated in advanced gastric cancer.

\begin{tabular}{|c|c|c|c|}
\hline No. & Accession no. & Gene symbol & Description \\
\hline 1 & NM_004190 & $L I P F$ & Lipase, gastric \\
\hline 2 & NM_020143 & PNO1 & Partner of NOB1 homolog (S. cerevisiae) \\
\hline 3 & NM_000257 & MYH7 & Myosin, heavy chain 7 , cardiac muscle, $\beta$ \\
\hline 4 & NM_015173 & $T B C 1 D 1$ & TBC1 (tre-2/USP6, BUB2, cdc16) domain family, member 1 \\
\hline 5 & NM_005408 & CCL13 & Chemokine (C-C motif) ligand 13 \\
\hline 6 & NM_174929 & ZMIZ2 & Zinc finger, MIZ-type containing 2 \\
\hline 7 & NM_004747 & $D L G 5$ & Discs, large homolog 5 (Drosophila) \\
\hline 8 & NM_024872.2 & $D O K 3$ & Docking protein 3 \\
\hline 9 & NM_201653 & CHIA & Chitinase, acidic \\
\hline 10 & NM_003893 & $L D B 1$ & LIM domain binding 1 \\
\hline 11 & NM_012455.2 & PSD4 & Pleckstrin and Sec7 domain containing 4 \\
\hline 12 & NM_005213 & CSTA & Cystatin A (stefin A) \\
\hline 13 & NM_005416 & $S P R R 3$ & Small proline-rich protein 3 \\
\hline 14 & NM_014989 & RIMS1 & Regulating synaptic membrane exocytosis 1 \\
\hline 15 & NM_001018005 & TPM1 & Tropomyosin $1(\alpha)$ \\
\hline 16 & NM_213589 & RAPH1 & Ras association (RalGDS/AF-6) and pleckstrin homology domains 1 \\
\hline 17 & NM_004898 & CLOCK & Clock homolog (mouse) \\
\hline 18 & NM_013292 & & Fast skeletal myosin light chain 2 \\
\hline 19 & NM_020321 & $A C C N 3$ & Amiloride-sensitive cation channel 3 \\
\hline 20 & NM_002754 & $M A P K 13$ & Mitogen-activated protein kinase 13 \\
\hline 21 & NM_013443 & ST6GALNAC6 & $\begin{array}{l}\text { ST6 ( } \alpha \text {-N-acetyl-neuraminyl-2,3- } \beta \text {-galactosyl-1,3)- } \\
\text { N-acetylgalactosaminide } \alpha \text {-2,6-sialyltransferase } 6\end{array}$ \\
\hline 22 & NM_001042453 & & Serine/threonine protein kinase MST4 \\
\hline 23 & NM_032646 & TTYH 2 & Tweety homolog 2 (Drosophila) \\
\hline 24 & NM_015089 & & p53-associated parkin-like cytoplasmic protein \\
\hline 25 & NM_003609 & HIRIP3 & HIRA interacting protein 3 \\
\hline 26 & NR_002219 & BIRC5 & Baculoviral IAP repeat-containing 5 (survivin) \\
\hline 27 & NM_000068 & CACNAIA & Calcium channel, voltage-dependent, P/Q type, $\alpha 1$ A subunit \\
\hline 28 & NM_203377 & $M B$ & Myoglobin \\
\hline 29 & NM_003768 & PEA15 & Phosphoprotein enriched in astrocytes 15 \\
\hline 30 & NM_053013 & ENO3 & Enolase $3(\beta$, muscle $)$ \\
\hline 31 & XR_018802 & $P I 4 K 2 A$ & Phosphatidylinositol 4-kinase type $2 \alpha$ \\
\hline 32 & NM_003725 & $H S D 17 B 6$ & Hydroxysteroid (17- $\beta$ ) dehydrogenase 6 homolog (mouse) \\
\hline 33 & NM_006063 & KBTBD10 & Kelch repeat and BTB (POZ) domain containing 10 \\
\hline 34 & NM_012288 & TRAM2 & Translocation associated membrane protein 2 \\
\hline 35 & NM_000730 & $C C K A R$ & Cholecystokinin A receptor \\
\hline 36 & NM_000290 & $P G A M 2$ & Phosphoglycerate mutase 2 (muscle) \\
\hline 37 & NM_199354 & PRB1 & Proline-rich protein BstNI subfamily 1 \\
\hline 38 & XR_019039 & $A C T B$ & Actin, $\beta$ \\
\hline 39 & NM_006478 & GAS2L1 & Growth arrest-specific 2 like 1 \\
\hline 40 & NM_024674 & LIN28 & Lin-28 homolog (C. elegans) \\
\hline 41 & NM_001070 & TUBG1 & Tubulin, $\gamma 1$ \\
\hline 42 & NM_015654 & NAT9 & N-acetyltransferase 9 \\
\hline 43 & NM_003643 & GCM1 & Glial cells missing homolog 1 (Drosophila) \\
\hline 44 & NM_006901.2 & MYO9А & Myosin IXA \\
\hline 45 & NM_017785 & CCDC 99 & Coiled-coil domain containing 99 \\
\hline 46 & NM_025135 & FHOD3 & Formin homology 2 domain containing 3 \\
\hline 47 & NM_022566 & MESDC1 & Mesoderm development candidate 1 \\
\hline 48 & NM_198255 & TERT & Telomerase reverse transcriptase \\
\hline 49 & NM_018231 & & Amino acid transporter \\
\hline 50 & NM_002458 & $M U C 5 B$ & Mucin 5B, oligomeric mucus/gel-forming \\
\hline 51 & NM_001001522 & $T A G L N$ & Transgelin \\
\hline
\end{tabular}


Table IV. Continued.

\begin{tabular}{|c|c|c|c|}
\hline No. & Accession no. & Gene symbol & Description \\
\hline 52 & NM_002631 & $P G D$ & Phosphogluconate dehydrogenase \\
\hline 53 & NM_006984 & CLDN10 & Claudin 10 \\
\hline 54 & NM_004359 & $C D C 34$ & Cell division cycle 34 homolog ( . cerevisiae) \\
\hline 55 & NM_001824 & $C K M$ & Creatine kinase, muscle \\
\hline 56 & NM_002274 & KRT13 & Keratin 13 \\
\hline 57 & XR_019039 & $A C T B$ & Actin, $\beta$ \\
\hline 58 & NM_000477 & $A L B$ & Albumin \\
\hline 59 & NM_001519.2 & $B R F 1$ & $\begin{array}{l}\text { BRF1 homolog, subunit of RNA polymerase III transcription } \\
\text { initiation factor IIIB ( } \text {. cerevisiae) }\end{array}$ \\
\hline 60 & NM_006790 & MYOT & Myotilin \\
\hline 61 & NM_021948 & $B C A N$ & Brevican \\
\hline 62 & NM_001142404.1 & $C D 164$ & CD164 molecule, sialomucin \\
\hline 63 & ВC050364.1 & C7orf13 & Chromosome 7 open reading frame 13 \\
\hline 64 & NM_005177 & ATP6V0A1 & ATPase, $\mathrm{H}^{+}$transporting, lysosomal V0 subunit a1 \\
\hline 65 & NM_020393 & PGLYRP4 & Peptidoglycan recognition protein 4 \\
\hline 66 & XM_937007 & FRMPD3 & FERM and PDZ domain containing 3 \\
\hline 67 & NM_024754 & PTCD2 & Pentatricopeptide repeat domain 2 \\
\hline 68 & NM_001098511 & $K I F 2 A$ & Kinesin heavy chain member $2 \mathrm{~A}$ \\
\hline 69 & NM_025058 & TRIM46 & Tripartite motif-containing 46 \\
\hline 70 & AK126458.1 & MYO15B & Myosin XVB pseudogene \\
\hline 71 & NM_018659 & CYTL1 & Cytokine-like 1 \\
\hline 72 & NM_002965 & S100A9 & S100 calcium binding protein A9 \\
\hline 73 & NM_032566 & SPINK7 & Serine peptidase inhibitor, Kazal type 7 (putative) \\
\hline 74 & NM_001669 & $A R S D$ & Arylsulfatase D \\
\hline 75 & NM_206820 & $M Y B P C 1$ & Myosin binding protein $\mathrm{C}$, slow type \\
\hline 76 & NM_003200 & $T C F 3$ & $\begin{array}{l}\text { Transcription factor } 3 \text { (E2A immunoglobulin enhancer } \\
\text { binding factors E12/E47) }\end{array}$ \\
\hline 77 & NM_031413 & CECR2 & Cat eye syndrome chromosome region, candidate 2 \\
\hline 78 & NM_017539 & DNAH3 & Dynein, axonemal, heavy chain 3 \\
\hline 79 & NM_017426 & NUP54 & Nucleoporin 54 kDa \\
\hline 80 & NM_002020 & FLT4 & Fms-related tyrosine kinase 4 \\
\hline 81 & NM_007320 & $R A N B P 3$ & RAN binding protein 3 \\
\hline 82 & NM_005286 & $N P B W R 2$ & Neuropeptides B/W receptor 2 \\
\hline 83 & NM_006428 & MRPL28 & Mitochondrial ribosomal protein L28 \\
\hline 84 & NM_014280.2 & DNAJC8 & DnaJ (Hsp40) homolog, subfamily C, member 8 \\
\hline 85 & NM_020679 & $M I F 4 G D$ & MIF4G domain containing \\
\hline 86 & NM_001823 & $C K B$ & Creatine kinase, brain \\
\hline 87 & NM_000477 & $A L B$ & Albumin \\
\hline 88 & NM_001927 & $D E S$ & Desmin \\
\hline 89 & NM_005416 & $S P R R 3$ & Small proline-rich protein 3 \\
\hline 90 & NM_022468 & $M M P 25$ & Matrix metallopeptidase 25 \\
\hline 91 & NM_016599 & MYOZ2 & Myozenin 2 \\
\hline 92 & NM_000243 & $M E F V$ & Mediterranean fever \\
\hline 93 & NM_002272 & KRT4 & Keratin 4 \\
\hline 94 & NM_003279 & $T N N C 2$ & Troponin C type 2 (fast) \\
\hline 95 & NM_006685 & $S M R 3 B$ & $\begin{array}{l}\text { Submaxillary gland androgen regulated protein } 3 \\
\text { homolog B (mouse) }\end{array}$ \\
\hline 96 & NM_014760 & TATDN2 & TatD DNase domain containing 2 \\
\hline 97 & NM_006928 & SILV & Silver homolog (mouse) \\
\hline 98 & NM_016522 & & Neurotrimin \\
\hline 99 & NM_000760 & CSF3R & Colony stimulating factor 3 receptor (granulocyte) \\
\hline 100 & NM_003167 & SULT2A1 & $\begin{array}{l}\text { Sulfotransferase family, cytosolic, 2A, } \\
\text { dehydroepiandrosterone (DHEA)-preferring, member } 1\end{array}$ \\
\hline
\end{tabular}


Table IV. Continued.

\begin{tabular}{|c|c|c|c|}
\hline No. & Accession no. & Gene symbol & Description \\
\hline 101 & NM_183360 & $D T N B$ & Dystrobrevin, $\beta$ \\
\hline 102 & NM_001711 & $B G N$ & Biglycan \\
\hline 103 & NM_023077 & Clorf163 & Chromosome 1 open reading frame 163 \\
\hline 104 & NM_015926.4 & TEX264 & Testis expressed 264 \\
\hline 105 & NM_006757 & TNNT3 & Troponin T type 3 (skeletal, fast) \\
\hline 106 & NM_002675 & $P M L$ & Promyelocytic leukemia \\
\hline 107 & XR_018113 & $G A P D H$ & Glyceraldehyde-3-phosphate dehydrogenase \\
\hline 108 & NM_021245 & MYOZ1 & Myozenin 1 \\
\hline 109 & NM_000383 & AIRE & Autoimmune regulator \\
\hline 110 & NM_006846 & SPINK5 & Serine peptidase inhibitor, Kazal type 5 \\
\hline 111 & XM_939725 & $A P 1 S 2$ & Adaptor-related protein complex 1 , sigma 2 subunit \\
\hline 112 & NM_024505 & NOX5 & NADPH oxidase, EF-hand calcium binding domain 5 \\
\hline 113 & NM_020145 & $S H 3 G L B 2$ & SH3-domain GRB2-like endophilin B2 \\
\hline 114 & NM_016192 & $T M E F F 2$ & $\begin{array}{l}\text { Transmembrane protein with EGF-like and two follistatin-like } \\
\text { domains } 2\end{array}$ \\
\hline 115 & NM_006472 & $T X N I P$ & Thioredoxin interacting protein \\
\hline 116 & NM_031866 & FZD8 & Frizzled homolog 8 (Drosophila) \\
\hline 117 & NM_003808 & TNFSF 13 & Tumor necrosis factor (ligand) superfamily, member 13 \\
\hline 118 & NM_015503 & $S H 2 B 1$ & SH2B adaptor protein 1 \\
\hline 119 & NM_014047 & C19orf53 & Chromosome 19 open reading frame 53 \\
\hline 120 & NM_022754 & SFXN1 & Sideroflexin 1 \\
\hline 121 & NM_003061 & SLIT1 & Slit homolog 1 (Drosophila) \\
\hline 122 & NM_003047 & SLC9A1 & $\begin{array}{l}\text { Solute carrier family } 9 \text { (sodium/hydrogen exchanger), member } 1 \\
\text { (antiporter, } \mathrm{Na}^{+} / \mathrm{H}^{+} \text {, amiloride sensitive) }\end{array}$ \\
\hline 123 & NM_021991 & $J U P$ & Junction plakoglobin \\
\hline
\end{tabular}

Table V. Positive ratio of the nine marker genes for detection of circulating gastric cancer cells.

\begin{tabular}{lccccccccc}
\hline Marker genes & TSPAN8 & EPCAM & HRH1 & CK20 & MMP12 & MMP7 & EGFR & REG3A & CEA \\
\hline Positive cases (\%) & 20 & 30 & 30 & 20 & 40 & 10 & 10 & 20 & 40 \\
\hline
\end{tabular}

Table VI. Number of positive genes in 10 cases by nested RT-PCR.

\begin{tabular}{lcccccccccc}
\hline Cases & GC-1 & GC-2 & GC-3 & GC-4 & GC-5 & GC-6 & GC-7 & GC-8 & GC-9 & GC-10 \\
\hline No. of positive genes & 1 & 0 & 2 & 3 & 5 & 6 & 1 & 2 & 0 & 2 \\
\hline
\end{tabular}

candidate genes, the expression of MMP12 and CEA mRNAs was positive in $40 \%$ of the patients with gastric cancer. However, the expression of the other seven genes was positive in $\leq 30 \%$ of the patients, respectively (Table V). We then investigated a combined effect of the expression of the nine genes on the detection of circulating cancer cells. Expression of one or more genes out of the nine was detected in $80 \%$ of the patients with gastric cancer by nested RT-PCR (Table VI).

We further investigated the association of the expression of the nine candidate marker genes with clinicopathological parameters of the 10 cases. We focused on four parameters: vascular invasion (v factor), lymphatic invasion (ly factor), lymph node metastasis ( $\mathrm{N}$ factor) and pathological stage I-IV, and investigated the association of these parameters with the total number of positive genes in the PBMCs of each patient (Fig. 1). Of the four parameters, the numbers of genes expressed in the PBMCs were $\leq 2$ in all of the vascular invasion-negative cases ( $\mathrm{v} 0$ ), while the numbers of genes were $\geq 2$ in 5 of 6 positive cases ( $v$ 1-3), exhibiting a significant difference between the two groups ( $\mathrm{P}=0.041$; Fig. $1 \mathrm{~A})$. However, no significant association was observed for the other three parameters (Fig. 1B-D), suggesting that the combined expression analysis of the nine marker genes using PBMCs detected micrometastasis through vascular invasion in the primary gastric cancer tissues. 

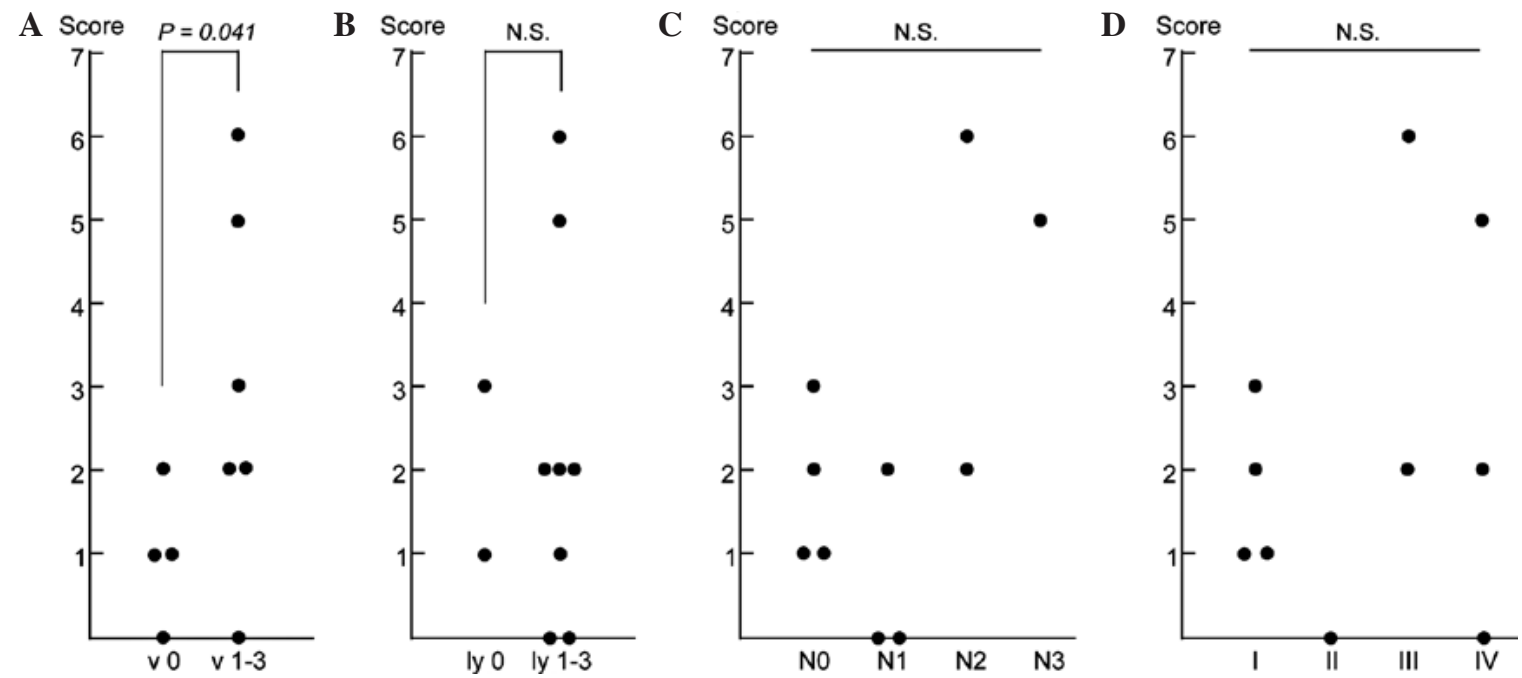

Figure 1. Relationships between clinicopathological parameters and the expression of the set of nine marker genes in PBMCs of gastric cancer patients. Associations of the total number of marker genes which were expressed in the PBMCs with (A) vascular invasion (v factor), (B) lymphatic invasion (ly factor), (C) lymph node metastasis ( $\mathrm{N}$ factor) and (D) pathological stage are shown. The score indicates the number of marker genes expressed in PBMCs of each patient. N.S., not significant $(\mathrm{P}>0.05)$. Student's t-test was used for A and B, and Cochran-Armitage test was used for C and D.

\section{Discussion}

Microarrays, at present, are widely used to analyze expression of thousands of genes simultaneously in cancer tissues. In the present study, we identified five genes (TSPAN8, EPCAM, $M M P 12, M M P 7$ and REG3A) as potential markers for the detection of circulating cancer cells in the peripheral blood of patients with gastric cancer through genome-wide gene expression profiling in combination with nested RT-PCR. Some of these genes have previously been reported to be up-regulated in gastric cancer cells; however, they have not previously been designated for the detection of circulating gastric cancer cells by nested RT-PCR. Furthermore, the combined expression analysis of the five genes and four previously reported marker genes, HRH1, EGFR, CK2O and CEA, revealed that one or more mRNAs among the nine genes could be detected in $80 \%$ of the patients with gastric cancer by nested RT-PCR, suggesting that a set of nine marker genes is more sensitive than a single marker gene for detection of circulating gastric cancer cells. In this study, we did not investigate the association of distant metastasis with expression of the nine marker genes since no patients had distant metastasis among the 10 studied patients. Although we could not exclude false-positive cases due to non-malignant epithelial cells which may have contaminated the blood samples during collection and which may have expressed the targeted transcripts (18), pathological $\mathrm{v}$ factor showed a significant association with the total number of marker genes expressed in the PBMCs of the patients. Hence, the set of nine marker genes may be promising for the detection of minimal amounts of circulating gastric cancer cells prior to the metastatic growth of gastric cancer cells in $\operatorname{organ}(\mathrm{s})$.

Among the five marker genes which were newly identified in the microarray analysis, we identified epithelial cell adhesion molecule (EPCAM) which is a member of a family of type I membrane proteins and pan-epithelial differentiation antigen expressed in many types of carcinomas (24-28).
Magnetic beads or structures coated with EPCAM monoclonal antibodies have been recently used for circulating cancer cell separation (29-31). Although we did not compare the accuracy of the detection of gastric cancer cells by these methods to that of nested RT-PCR since we did not conduct the former assays, $30 \%$ of patients with gastric cancer exhibited EPCAM-positivity in PBMCs by nested RT-PCR. Further clinical study investigating the relationship between the clinical outcome of patients and EPCAM expression in PBMCs by nested RT-PCR may clarify whether this method could be clinically applied for the detection of circulating gastric cancer cells. Two matrix metalloproteinases, MMP7 and $M M P 12$, were among the five marker genes which were newly identified in this study. MMPs are a family of zinc-dependent proteolytic enzymes capable of cleaving extracellular matrix proteins, and the expression of MMPs in cancer tissue has been reported to be associated with the risk of metastasis (32-38). These two MMPs may play important roles in tumor invasion and the formation of metastasis in gastric cancer.

In conclusion, five novel marker genes were designated for the detection of circulating gastric cancer cells. The nested RT-PCR analysis for the set of nine marker genes, TSPAN8, EPCAM, MMP12, MMP7, REG3A, HRH1, EGFR, CK2O and $C E A$, using PBMCs of patients with gastric cancer may provide the potential for the detection of circulating gastric cancer cells prior to the formation of metastasis in other organs. Our data suggest that early detection and personalized therapy for gastric cancers, by prescribing the appropriate treatment to patients with a high risk of metastasis, may be achievable by utilizing specific sets of marker genes according to the approach shown here.

\section{Acknowledgements}

We thank Tomohisa Furuhata, Yasutoshi Kimura, Chikashi Kihara, Kenji Okita and Noriko Nishikawa for the helpful discussions. 


\section{References}

1. Thun MJ, DeLancey JO, Center MM, Jemal A and Ward EM: The global burden of cancer: priorities for prevention. Carcinogenesis 31: 100-110, 2009.

2. Ott K, Lordick F, Blank S and Buchler M: Gastric cancer: surgery in 2011. Langenbecks Arch Surg: Jan. 14, 2011 (E-pub ahead of print).

3. Chen XM, Chen GY, Wang ZR, Zhu FS, Wang XL and Zhang X: Detection of micrometastasis of gastric carcinoma in periphera blood circulation. World J Gastroenterol 10: 804-808, 2004.

4. Joyce JA and Pollard JW: Microenvironmental regulation of metastasis. Nat Rev Cancer 9: 239-252, 2009.

5. Vardakis N, Messaritakis I, Papadaki C, et al: Prognostic significance of the detection of peripheral blood CEACAM5 mRNA-positive cells by real-time polymerase chain reaction in operable colorectal cancer. Clin Cancer Res 17: 165-173, 2011.

6. Miyazono F, Natsugoe S, Takao S, et al: Surgical maneuvers enhance molecular detection of circulating tumor cells during gastric cancer surgery. Ann Surg 233: 189-194, 2001.

7. Pantel K, Brakenhoff RH and Brandt B: Detection, clinical relevance and specific biological properties of disseminating tumour cells. Nat Rev Cancer 8: 329-340, 2008.

8. Qiu MZ, Li ZH, Zhou ZW, et al: Detection of carcinoembryonic antigen messenger RNA in blood using quantitative real-time reverse transcriptase-polymerase chain reaction to predict recurrence of gastric adenocarcinoma. J Transl Med 8: 107, 2010.

9. Guadagni F, Kantor J, Aloe S, et al: Detection of blood-borne cells in colorectal cancer patients by nested reverse transcription-polymerase chain reaction for carcinoembryonic antigen messenger RNA: longitudinal analyses and demonstration of its potential importance as an adjunct to multiple serum markers. Cancer Res 61: 2523-2532, 2001

10. Ikeguchi M and Kaibara N: Detection of circulating cancer cells after a gastrectomy for gastric cancer. Surg Today 35: 436-441, 2005.

11. Dardaei L, Shahsavani R, Ghavamzadeh A, et al: The detection of disseminated tumor cells in bone marrow and peripheral blood of gastric cancer patients by multimarker (CEA, CK20, TFF1 and MUC2) quantitative real-time PCR. Clin Biochem 44: 325-330, 2011

12. Katsuragi K, Yashiro M, Sawada T, Osaka H, Ohira M and Hirakawa K: Prognostic impact of PCR-based identification of isolated tumour cells in the peritoneal lavage fluid of gastric cancer patients who underwent a curative $\mathrm{R} 0$ resection. $\mathrm{Br} J$ Cancer 97: 550-556, 2007.

13. Mori K, Aoyagi K, Ueda T, et al: Highly specific marker genes for detecting minimal gastric cancer cells in cytology negative peritoneal washings. Biochem Biophys Res Commun 313: 931-937, 2004

14. Zembutsu H, Ohnishi Y, Daigo Y, et al: Gene-expression profiles of human tumor xenografts in nude mice treated orally with the EGFR tyrosine kinase inhibitor ZD1839. Int J Oncol 23: 29-39, 2003.

15. Zembutsu H, Ohnishi Y, Tsunoda T, et al: Genome-wide cDNA microarray screening to correlate gene expression profiles with sensitivity of 85 human cancer xenografts to anticancer drugs. Cancer Res 62: 518-527, 2002

16. Zembutsu H, Suzuki Y, Sasaki A, et al: Predicting response to docetaxel neoadjuvant chemotherapy for advanced breast cancers through genome-wide gene expression profiling. Int J Oncol 34: 361-370, 2009 .

17. Zembutsu H, Yanada M, Hishida A, et al: Prediction of risk of disease recurrence by genome-wide cDNA microarray analysis in patients with Philadelphia chromosome-positive acute lymphoblastic leukemia treated with imatinib-combined chemotherapy. Int J Oncol 31: 313-322, 2007.

18. Obermayr E, Sanchez-Cabo F, Tea MK, et al: Assessment of a six gene panel for the molecular detection of circulating tumor cells in the blood of female cancer patients. BMC 10: 666, 2010.

19. Luo L, Salunga RC, Guo H, et al: Gene expression profiles of laser-captured adjacent neuronal subtypes. Nat Med 5: 117-122, 1999.
20. Saito-Hisaminato A, Katagiri T, Kakiuchi S, Nakamura T, Tsunoda T and Nakamura Y: Genome-wide profiling of gene expression in 29 normal human tissues with a cDNA microarray. DNA Res 9: 35-45, 2002.

21. Hasegawa S, Furukawa Y, Li M, et al: Genome-wide analysis of gene expression in intestinal-type gastric cancers using a complementary DNA microarray representing 23,040 genes. Cancer Res 62: 7012-7017, 2002.

22. Amin AT, Shiraishi N, Ninomiya S, Tajima M, Inomata M and Kitano S: Increased mRNA expression of epidermal growth factor receptor, human epidermal receptor, and survivin in human gastric cancer after the surgical stress of laparotomy versus carbon dioxide pneumoperitoneum in a murine model. Surg Endosc 24: 1427-1433, 2010.

23. Mori M, Mimori K, Inoue H, et al: Detection of cancer micrometastases in lymph nodes by reverse transcriptase-polymerase chain reaction. Cancer Res 55: 3417-3420, 1995.

24. Trebak M, Begg GE, Chong JM, Kanazireva EV, Herlyn D and Speicher DW: Oligomeric state of the colon carcinomaassociated glycoprotein GA733-2 (Ep-CAM/EGP40) and its role in GA733-mediated homotypic cell-cell adhesion. J Biol Chem 276: 2299-2309, 2001.

25. Maghzal N, Vogt E, Reintsch W, Fraser JS and Fagotto F: The tumor-associated EpCAM regulates morphogenetic movements through intracellular signaling. J Cell Biol 191: 645-659, 2010.

26. Du W, Ji H, Cao S, et al: EpCAM: a potential antimetastatic target for gastric cancer. Dig Dis Sci 55: 2165-2171, 2009.

27. Kuhn S, Koch M, Nubel T, et al: A complex of EpCAM, claudin-7, CD44 variant isoforms, and tetraspanins promotes colorectal cancer progression. Mol Cancer Res 5: 553-567, 2007.

28. Mukherjee S, Richardson AM, Rodriguez-Canales J, et al: Identification of EpCAM as a molecular target of prostate cancer stroma. Am J Pathol 175: 2277-2287, 2009.

29. Sha MY, Xu H, Natan MJ and Cromer R: Surface-enhanced Raman scattering tags for rapid and homogeneous detection of circulating tumor cells in the presence of human whole blood. J Am Chem Soc 130: 17214-17215, 2008.

30. Myung JH, Launiere CA, Eddington DT and Hong S: Enhanced tumor cell isolation by a biomimetic combination of E-selectin and anti-EpCAM: implications for the effective separation of circulating tumor cells (CTCs). Langmuir 26: 8589-8596, 2010.

31. Hosokawa M, Hayata T, Fukuda Y, et al: Size-selective microcavity array for rapid and efficient detection of circulating tumor cells. Anal Chem 82: 6629-6635, 2010.

32. Shi WD, Meng ZQ, Chen Z, Lin JH, Zhou ZH and Liu LM: Identification of liver metastasis-related genes in a novel human pancreatic carcinoma cell model by microarray analysis. Cancer Lett 283: 84-91, 2009.

33. Szarvas T, Becker M, vom Dorp F, et al: Matrix metalloproteinase-7 as a marker of metastasis and predictor of poor survival in bladder cancer. Cancer Sci 101: 1300-1308, 2010.

34. Oshima T, Akaike M, Yoshihara K, et al: Clinicopathological significance of the gene expression of matrix metalloproteinase-7, insulin-like growth factor-1, insulin-like growth factor-2 and insulin-like growth factor-1 receptor in patients with colorectal cancer: insulin-like growth factor-1 receptor gene expression is a useful predictor of liver metastasis from colorectal cancer. Oncol Rep 20: 359-364, 2008.

35. Fang YJ, Lu ZH, Wang GQ, et al: Elevated expressions of MMP7, TROP2, and survivin are associated with survival, disease recurrence, and liver metastasis of colon cancer. Int $\mathrm{J}$ Colorectal Dis 24: 875-884, 2009.

36. Kerkela E, Ala-aho R, Klemi P, et al: Metalloelastase (MMP-12) expression by tumour cells in squamous cell carcinoma of the vulva correlates with invasiveness, while that by macrophages predicts better outcome. J Pathol 198: 258-269, 2002.

37. Balaz P, Friess H, Kondo Y, Zhu Z, Zimmermann A and Buchler MW: Human macrophage metalloelastase worsens the prognosis of pancreatic cancer. Ann Surg 235: 519-527, 2002.

38. Hofmann HS, Hansen G, Richter G, et al: Matrix metalloproteinase-12 expression correlates with local recurrence and metastatic disease in non-small cell lung cancer patients. Clin Cancer Res 11: 1086-1092, 2005. 